\title{
Estimación de la amenaza de inundación a partir de la comparación de caudales máximos en la parte alta del Río Frío en el Municipio de Zipaquirá, Cundinamarca
}

\section{Estimation of the threat of flooding from the comparison of maximum flows in the upper part of the municipality of Zipaquirá, Cundinamarca}

\section{Paula Andrea Salamanca Urrego ${ }^{1}$, María Alejandra Ruge Vera ${ }^{2}$, Jesús Ernesto Torres Quintero ${ }^{3}$}

1. Ingeniería Ambiental, Universidad Libre. paulaa.salamancau@unilibrebog.edu.co

2 Ingeniería Ambiental, Universidad Libre.mariaa.rugev@unilibrebog.edu.co

${ }^{3}$ Docente investigador, Universidad Libre. jesuse.torresq@unilibrebog.edu.co

\section{Resumen}

Esta investigación tiene como objetivo estimar la amenaza de inundación a partir de la comparación de caudales máximos. El trabajo inicia con la determinación de las características del río, el análisis de la cobertura vegetal, y posteriormente el cálculo de los caudales máximos por Método Racional y el Análisis de Frecuencia Hidrológica, terminando con la determinación de los diferentes niveles de inundación por medio del modelo Manning. El enfoque de la amenaza de inundación se dará a partir modelo Manning, utilizando los caudales máximos para cada periodo de retorno 10-25-100 años. A lo largo del río de estudio se realizaron 21 secciones trasversales, las cuales demostraron después de su procesamiento una alta probabilidad de inundación de las secciones de la 1-10 y poca probabilidad de inundación en las secciones restantes. Cabe resaltar que no se ha realizado ningún estudio hasta el momento para estimar la amenaza de inundación en dicha zona y que es de gran importancia hacerlo pues podría ser un gran aporte para prevenir futuras inundaciones, siendo una herramienta significativa para el POT de Zipaquirá y Tabio, Cundinamarca. Además es una contribución importante para la sostenibilidad de Zipaquirá, según objetivo 11: Ciudades Sostenibles, de los objetivos del Desarrollo Sostenible de la ONU.
Palabras clave: análisis de frecuencia hidrológica, caudal, niveles de inundación.

\begin{abstract}
The objective of this research is to estimate the threat of flooding by comparing the maximum levels in the upper part of the Río Frío in Zipaquirá, Cundinamarca. The work started with the determination of the characteristics of the river, the analysis of the vegetation cover, and the subsequent calculation of the lowest levels by the rational method and the hydrological frequency analysis, ending with the determination of the different flood levels by half of the Manning model. The focus of the flood threat will be based on the Manning model, using the maximum flow rates for each return period 10-25-50-100 years. The results reveal that in most of the sections the water level does not exceed the level of the river, however in some areas it does. It should be noted that no study has been conducted so far to estimate the threat of flooding in this area and that it is of great importance to do so because it could be a great contribution to prevent future flooding, being a significant tool for the POT of Zipaquirá and Tabio, Cundinamarca. It is also an important contribution to the sustainability of Zipaquirá, according to the objective: Sustainable Cities, of the objectives of the Sustainable Development of the UN.
\end{abstract}


49

Keywords: analysis of hydrological frequency, flow, flood levels.

\section{Introducción}

La Niña (frío) y el Niño (cálido) corresponden a fenómenos señal de la variabilidad climática en la franja tropical del océano pacífico, según la aparición de aguas superficiales relativamente cálidas (El Niño) o más frías (La Niña), dichos fenómenos se manifiestan en áreas de la superficie terrestre como en el territorio colombiano, una revelación de esto se evidenció en 2010-2011, por el fenómeno de "la niña" el cual trascendió en el clima nacional, pues generó accidentes por crecientes súbitas, pérdidas humanas y materiales.

(SIAC,

2014)

Zipaquirá es un municipio que se ubica en el norte de Cundinamarca, Colombia, cuenta con una precipitación promedio de $805 \mathrm{~mm}$ al año entre los meses más húmedos (Alcaldía Zipaquirá, 2013), teniendo en cuenta esto, y el alza de la población en el municipio que genera aumento de urbanizaciones y con esto la inhabilitación del drenaje natural de agua, se establece la alta posibilidad de amenaza de inundación según el Análisis de Gestión de Riesgo y Desastre de Colombia, en el que se realizó registro continuo de las estaciones hidrometeorológicas. (UNGRD, 2016)

El área de estudio se ubica específicamente en el norte del municipio de Zipaquirá, en la vereda de Páramo Guerrero, San Isidro y Ventalarga, en las que se ubica Río Frío. En la figura 1 se observa la localización de dicha área, asimismo las estaciones hidrometeorológicas: Páramo Guerrero, Ventalarga y Pozo Hondo, las cuales se utilizan en la presente investigación.

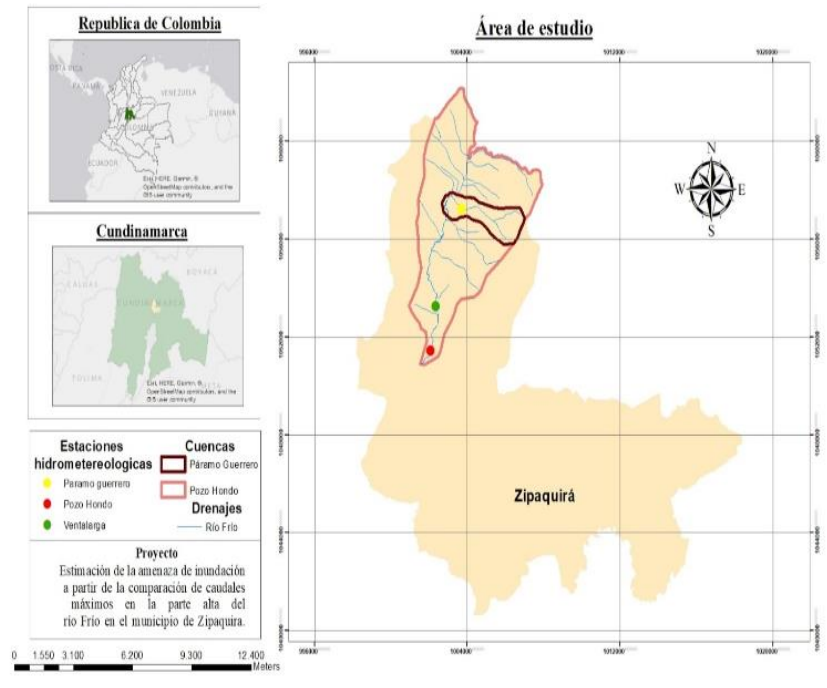

Figura 1. Área de Estudio

\section{Metodología}

En la estimación de la amenaza de inundación se requieren técnicas/instrumentos para la obtención de datos, en este trabajo se utiliza el método estadístico; Análisis de Frecuencia Hidrológica, y el Método Racional, para el cálculo de los Q.máx. Así como se usa el modelo Manning en el cálculo de los niveles de inundación. El grupo de investigación clasificó el proyecto en III etapas según cada objetivo del proyecto.

\subsection{Determinación de características y parámetros fisiográficos del Río Frío}

Las fuentes de información en términos de caudales, precipitación, y topografía de la zona, requieren la interacción de instituciones como el IGAC, 2015, que permite la obtención de drenajes, cobertura vegetal, la CAR, 2015 recoge los datos de precipitación y caudales, el Portal Vertex, 2012 aporta información geoespacial en 
el procesamiento de las curvas de nivel. Para el cálculo del área, la pendiente, así como la determinación de la cobertura vegetal se realiza un procesamiento en el software ArcGis10.4

\subsection{Obtención y comparación de caudales máximos}

Las curvas IDF son determinantes para el cálculo de la intensidad de precipitación máxima en 24horas de cada estación (Monsalve, 1997). Dicha intensidad se utiliza en la obtención del Q.máx en los diferentes TR (tiempos de retorno) de 10-25-100 años de cada estación a partir del Método Racional $\left(\mathrm{Q}=\mathrm{C}^{*} \mathrm{I}^{*} \mathrm{~A}\right)$, involucrando su escorrentía (C) según la cobertura vegetal de la zona, y área de la cuenca. Asimismo se calcula el Q.máx a partir de modelo SMADA (distribución Gumbel) y se usa el software HYFA (distribución-Log.Pearson). (Campos, 2001)

Finalmente, se comparan y calcula el promedio para los Q.máx obtenidos por los dos métodos mencionados anteriorente, generando un único Q.máx para cada uno de los TR.

\subsection{Determinación de niveles de inundación y estimación de la amenaza}

Las batimetrías requieren la medición de secciones transversales, determinan la elevación del terreno y el río. Dichos datos son utilizados en la ecuación Manning para la elaboración de una gráfica que involucra los diferentes niveles del río, el área y su radio hidráulico, tal gráfica permite entrar con los datos obtenidos de cada Q.máx, y determinar así los diferentes niveles de inundación para $\mathrm{TR}=10,25,100$ años. (Torres, 2018)

Resueltamente, según los niveles identificados se establecen las áreas inundables basándose en la ocurrencia del evento en determinado tiempo.
50

En el cálculo del Q.máx por el Método Racional, primero se realizó el cálculo de la intensidad que depende de la longitud del río y la pendiente, con los cuales se determinan los tiempos de concentración y con estos la intensidad de cada TR. En el caso del C se identificaron: bosquescultivos-pastos según las dos áreas. Finalmente en el método racional se demostró que el $\mathrm{Q}$ en Ventalarga es mucho mayor que P.Guerrero, teniendo en cuenta su área. A continuación se muestran los cálculos:

Tabla 1. Comparación Método Racional

\begin{tabular}{|c|c|c|c|c|}
\hline \multicolumn{5}{|c|}{ Estación-P.Guerrero } \\
\hline & $\mathbf{C}$ & $\mathbf{I}(\mathbf{m} / \mathbf{s})$ & $\mathbf{A}(\mathbf{m})$ & $\mathrm{Q}(\mathrm{m} 3 / \mathrm{s})$ \\
\hline 10 & 0,43 & \multirow{3}{*}{0,0001} & \multirow{3}{*}{4400000} & 13,483 \\
\hline 25 & 0,47 & & & 28,542 \\
\hline 100 & 0,534 & & & 62,398 \\
\hline \multicolumn{5}{|c|}{ Estación-Ventalarga } \\
\hline 10 & 0,43 & \multirow{3}{*}{0,0001} & \multirow{3}{*}{37800000} & 70,42 \\
\hline 25 & 0,47 & & & 90,869 \\
\hline 100 & 0,53 & & & 133,452 \\
\hline
\end{tabular}

Al calcular el Q.máx por el Análisis de Frecuencia Hidrológica, se utilizó programa HYFA y el modelo SMADA, para las áreas de las estaciones P.Guerrero y P.Hondo (en vez de Ventalarga, pues no existe estación de caudales Ventalarga, pero sí una estación cercana; Pozo Hondo). Seguidamente se muestran los resultados:

Tabla 2. Comparación Método Estadístico

\begin{tabular}{|c|c|c|c|}
\hline \multicolumn{4}{|c|}{ Pozo-Hondo } \\
\hline TR & Gumbel & Log-Pearson & Promedio \\
\hline $\mathbf{1 0}$ & 37,17 & 35,419 & 36,29 \\
\hline $\mathbf{2 5}$ & 47,121 & 49,534 & 48,3279 \\
\hline $\mathbf{1 0 0}$ & 61,83 & 76,563 & 69,1965 \\
\hline \multicolumn{4}{|c|}{ Páramo-Guerrero } \\
\hline $\mathbf{1 0}$ & 21,43 & 15,343 & 18,3865 \\
\hline $\mathbf{2 5}$ & 29,25 & 31,65 & 30,45 \\
\hline $\mathbf{1 0 0}$ & 40,8 & 90,704 & 65,752 \\
\hline
\end{tabular}

Según lo anterior, se evidencian valores cercanos

\section{Resultados}


51

por los 2 métodos para el área de P.Guerrero, sin embargo para P.Hondo y Ventalarga sus resultados tienen gran variación. Para el cálculo de los niveles de inundación por Manning, se establecieron 21 secciones.

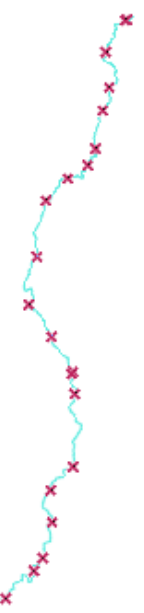

Figura 2. Río Frío con Batimetría

Dependiendo del borde mínimo de cada una de las secciones trasversales, se mide la profundidad, dividiendo en 6 partes iguales, con el fin de obtener los datos que serán usados para determinar los niveles de inundación por la ecuación de Manning.

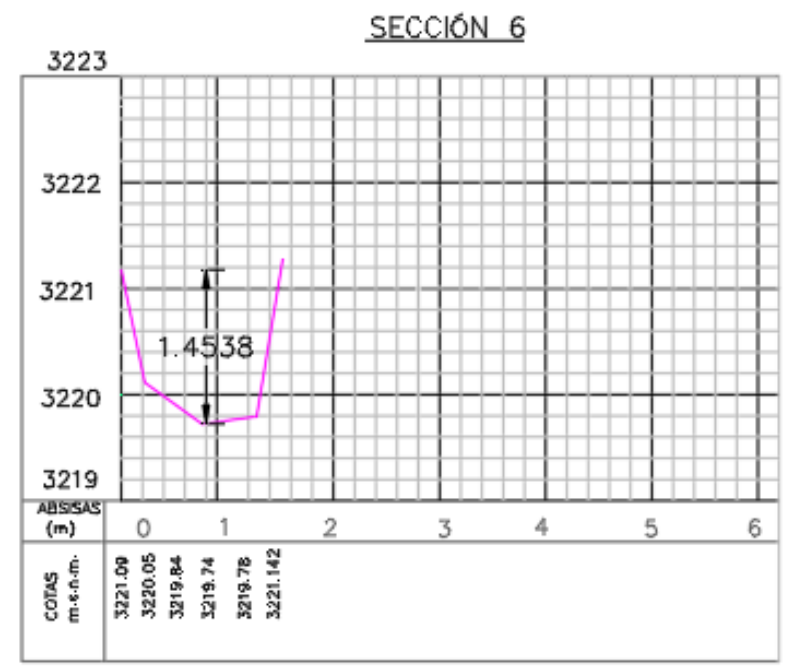

Figura 3. Sección 6 Río Frío

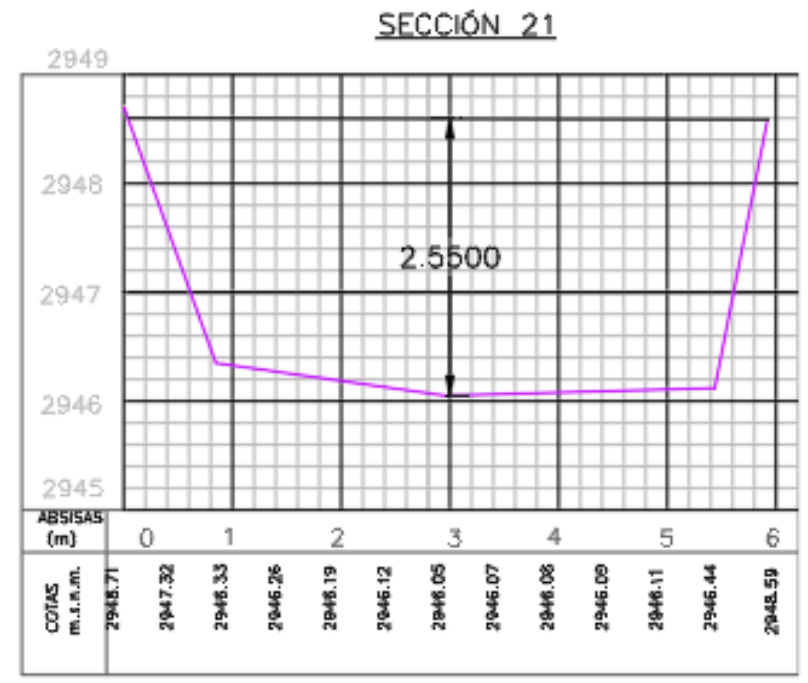

Figura 4. Sección 21 Río Frío

Con los datos obtenidos se realizan gráficas de

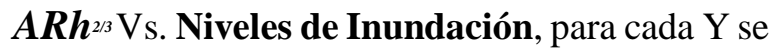
calcula su respectivo $A R h^{2 / 3}$, posterior a esto se tiene en cuenta que $A R h^{2 / 3}=\left(\mathrm{Q}^{*} \mathrm{n}\right) /\left(\mathrm{S}^{1 / 2}\right)$, y se calcula dicho dato teniendo en cuenta los Q.máx para cada TR, el factor de fricción de Manning y su respectiva pendiente. Finalmente, se entra a la gráfica mencionada con un nuevo valor de $A R h^{2 / 3}$, determinando así su nivel de inundación Y. 


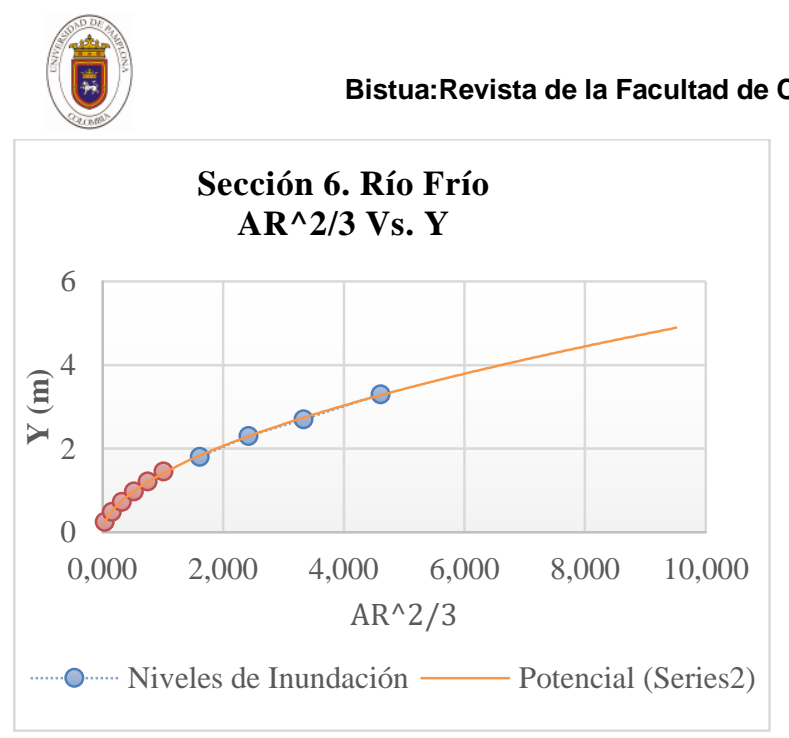

Figuras 5. Gráficos $A R h^{2 / 3}$ Vs. Y Sección 6

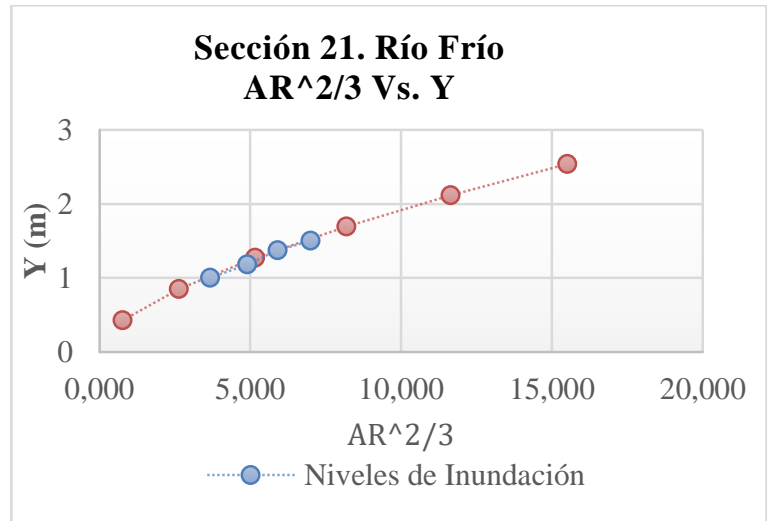

Figuras 6. Gráficos $A R h^{2 / 3}$ Vs. Y Sección 21

Se evidencia desbordamiento para la sección 6, debido a que los niveles del río para $\mathrm{TR}=10-25$ 100 años superan los bordes del río, a diferencia de la sección 21 en donde no se presenta desbordamiento.

Las profundidades de inundación obtenidas anteriormente se grafican en cada una de las secciones, para determinar la amenaza alta (rojo), media (amarillo) o baja (verde).
SECCIONN 6

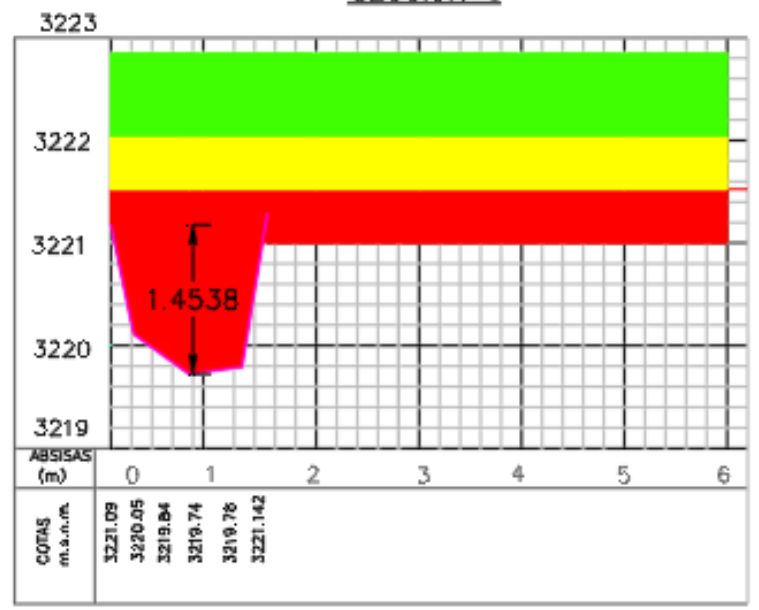

Figura 7. Niveles de Inundación Sección 6

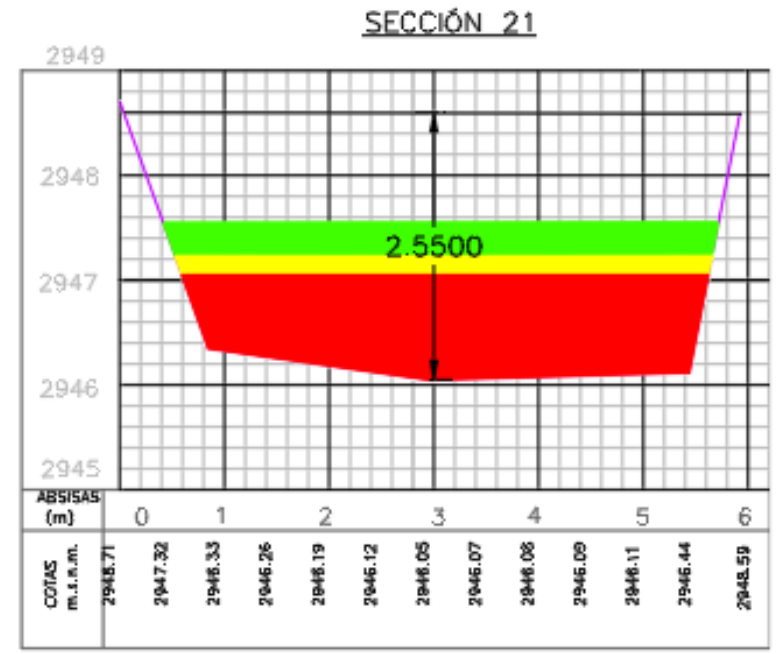

Figura 8. Niveles de Inundación Sección 21

Dentro de las 21 secciones, de la 1-6 tienen una alta amenaza de inundación, puesto que el TR para 10 años ya sobrepasa el borde mínimo de las secciones. La secciones 7-8 tienen amenaza media y las demás secciones no cuentan con amenaza de inundación, así como se muestra en la sección 21. 
53

\section{Conclusiones}

Se determinaron los Q.máx para TR en el área de las estaciones P.Guerrero, P.Hondo y Ventalarga, realizando la comparación entre el Método Racional y Análisis de Frecuencia Hidrológica, evidenciando que el primero es más usado en cuencas de pequeñas áreas y el segundo en toda clase de cuencas.

Asimismo se establecieron los diferentes niveles de inundación según Manning, determinando la altura del agua a 10-25-100 años, concluyendo que las secciones 1-10 cuentan con amenaza alta de inundación, puesto que superan el borde de dichas secciones, es importante tener en cuenta los anteriores cálculos en el POT de Zipaquirá y Tabio, sabiendo que son áreas cercanas a una escuela rural, casas y cultivos.

Se plantea realizar un modelamiento hidrológico con el Software HecRas5.0.3 para establecer claramente las áreas susceptibles a ser inundadas.

\section{Agradecimientos}

A Dios, quien diariamente ha sido el motor fundamental en nuestras vidas. Al Ing. Jesús Ernesto Torres y a la Ing. Lizeth Vanessa Salamanca Urrrego por todo el apoyo en el desarrollo de la investigación. A todos los docentes de la Universidad Libre quienes aportaron con sus conocimientos, fortalecieron e hicieron de esta una mejor investigación.

\section{Refrencias Bibliografícas}

Chow V. (1997). Hidráulica para canales Abiertos. San Francisco: McGraw Hill. Monsalve, G. (1995). Hidráulica en la Ingeniería. Escuela Colombiana de Ingeniería.
INVIAS. (2013). Manual de drenaje Invias. Recuperado de https://www.invias.gov.co/index.php/arc hivo-y-documentos/documentostecnicos/especificaciones-tecnicas/984$\underline{\text { manual-de-drenaje-para-carreteras }}$

Rajasekar, U., Ramirez, J.A., Dhruvesg, P.P, Coulthard, T.J., (2016) Flood modeling can make a difference. Earth Syst. Sci. http://dx.doi.org/10.5194/hess-2016-544.

Mosquera, J., Carvajal, E, (2011) La gestión del riesgo-de la incertidumbre a la adaptabilidad. Revista Bistua. ISSN 0120-4211.

http://www.redalyc.org:9081/home.oa?ci $\mathrm{d}=1666661$

J.E. Torres Quintero, Ingeniero Civil de la Universidad La Gran Colombia, Especialista en hidrología general y aplicada del Consejo Superior de Investigaciones Científicas CSIC, Magister en Recursos hidráulicos de la Universidad Nacional de Colombia, Bogotá. Profesor Asociado de la Universidad Libre de Colombia, Bogotá, Facultad de Ingeniería; Integrante activo del Grupo de Investigación Tecnoambiental de la Universidad Libre de Colombia, Bogotá.

*Para citar este artículo: Salamanca Urrego PA.; Ruge Vera MA.; Jesús Ernesto Torres Quintero JE. Estimation of the threat of flooding from the comparison of maximum flows in the upper part of the municipality of Zipaquirá, Cundinamarca

+ Autor para el envió de correspondencia y la solicitud de las separatas: Salamanca Urrego PA.Universidad Libre. paulaa.salamancau@unilibrebog.edu.co

Recibido: Octubre 24 de 2016

Aceptado: Enero 31 de 2017 
